\title{
High-temperature ferroelectric order and magnetoelectric coupling driven by the magnetic field cooling effect in $R_{2} \mathrm{BaCuO}_{5}(R=\mathrm{Er}, \mathrm{Dy}, \mathrm{Sm})$
}

\author{
A. Indra, ${ }^{1,2}$ S. Mukherjee,,${ }^{1}$ S. Majumdar, ${ }^{1}$ O. Gutowski, ${ }^{3}$ M. v. Zimmermann, ${ }^{3}$ and S. Giri ${ }^{1, *}$ \\ ${ }^{1}$ School of Physical Sciences, Indian Association for the Cultivation of Science, Jadavpur, Kolkata 700032, India \\ ${ }^{2}$ Department of Physics, Srikrishna College, Bagula, Nadia, West Bengal, 741502, India \\ ${ }^{3}$ Deutsches Elektronen-Synchrotron DESY, Notkestr. 85, 22603 Hamburg, Germany
}

(Received 15 April 2019; published 10 July 2019)

\begin{abstract}
The high-temperature ferroelectric order and a remarkable magnetoelectric effect driven by the magnetic field cooling are reported in $R_{2} \mathrm{BaCuO}_{5}(R=\mathrm{Er}, \mathrm{Dy}, \mathrm{Sm})$ series. The ferroelectric (FE) orders are observed at much higher temperatures than their magnetic orders for all three members. The value of FE Curie temperature $\left(T_{\mathrm{FE}}\right)$ is considerably high as $\sim 235 \mathrm{~K}$ with the polarization value $(P)$ of $\sim 1410 \mu \mathrm{C} / \mathrm{m}^{2}$ for a $4 \mathrm{kV} / \mathrm{cm}$ poling field in the case of $\mathrm{Er}_{2} \mathrm{BaCuO}_{5}$, whereas the values of $T_{\mathrm{FE}}$ and $P$ are also promising as $\sim 232$ and $\sim 992 \mu \mathrm{C} / \mathrm{m}^{2}$ for $\mathrm{Dy}_{2} \mathrm{BaCuO}_{5}$, and $\sim 184 \mathrm{~K}$ and $\sim 980 \mu \mathrm{C} / \mathrm{m}^{2}$ for $\mathrm{Sm}_{2} \mathrm{BaCuO}_{5}$. The synchrotron diffraction studies of $\mathrm{Dy}_{2} \mathrm{BaCuO}_{5}$ confirm a structural transition at $T_{\mathrm{FE}}$ to a polar $P n a 2_{1}$ structure, which correlates the FE order. An unusual magnetoelectric coupling is observed below the $R$ order for Er and Dy compounds and below the $\mathrm{Cu}$ order for $\mathrm{Sm}$ compound, when the pyroelectric current is recorded only with the magnetic field both in heating and cooling cycles, i.e., the typical magnetic field cooled effect. The magnetic field cooled effect driven emergence of polarization is ferroelectric in nature, as it reverses due to the opposite poling field.
\end{abstract}

DOI: 10.1103/PhysRevB.100.014413

\section{INTRODUCTION}

Multiferroics, where ferroelectric order coexist with the long range magnetic order, attract special attention for the magnetoelectric (ME) cross coupling [1-3]. In addition to the fundamental interest on the origin of coexisting $\mathrm{ME}$ orders in a chemically single phase compounds, multiferroics are extremely promising for the applications $[1,4-6]$. For the practical applications, the occurrence of ME orders associated with the strong ME coupling is highly desirable, which is still missing except for very few promising inorganic materials like $\mathrm{BiFeO}_{3}$ [7,8], $\mathrm{CuO}$ [9], and $\mathrm{Sr}_{3} \mathrm{Co}_{2} \mathrm{Fe}_{24} \mathrm{O}_{41}$ [10]. Herein, the occurrence of the ferroelectric (FE) order is revealed at significantly high temperature in an unexplored $R_{2} \mathrm{BaCuO}_{5}(R=\mathrm{Er}$, Dy, Sm) series. For example, the FE order is observed around $\sim 235 \mathrm{~K}$ associated with a large value of electric polarization $(P)$ of $\sim 1410 \mu \mathrm{C} / \mathrm{m}^{2}$ for a $4 \mathrm{kV} / \mathrm{cm}$ poling field (E) in the case of $\mathrm{Er}_{2} \mathrm{BaCuO}_{5}$. Furthermore, an intriguing magnetic field cooled effect driven strong ME coupling is observed below the magnetic ordering temperature in the $R_{2} \mathrm{BaCuO}_{5}$ series.

The $R_{2} \mathrm{BaCuO}_{5}$ series of compounds crystallize in the Pnma $(\mathrm{Z}=4)$ space group, where the copper ions are occupied in the distorted square pyramids $\left(\mathrm{CuO}_{5}\right)$ and are connected by the $\mathrm{RO}_{7}$ polyhedra [11-14]. Direct linkages between $\mathrm{RO}_{7}$ and linkage through $\mathrm{CuO}_{5}$ are depicted in Fig. 1. It has been observed that the lattice parameters and volume of the unit cell decrease linearly as a function of the lanthanide ionic radius starting from $\mathrm{Sm}^{3+}$ to $\mathrm{Lu}^{3+}$.[14] The short-range force constant model was used for interpreting the results of Raman and infrared wave numbers in the

*Corresponding author: sspsg2@iacs.res.in orthorhombic phase of $R_{2} \mathrm{BaCuO}_{5}(R=\mathrm{Y}$, Ho, Gd), which was consistent with the orthorhombic phase of Pnma space group [15]. The heat capacity [16] and magnetization results $[17,18]$ confirmed two magnetic transitions corresponding to antiferromagnetic (AFM) $\mathrm{Cu}^{2+}$ and $R^{3+}$ orders, respectively. The electron paramagnetic resonance spectra were recorded for a few members of the $R_{2} \mathrm{BaCuO}_{5}$ series, where a strong exchange coupling was suggested between $\mathrm{Cu}^{2+}$ and $R^{3+}$ ions [19]. In fact, significant $\mathrm{Cu}^{2+}$ and $R^{3+}$ exchange interaction and large magnetic anisotropy were proposed from the magnetic and spectroscopic investigations for $R=$ Dy and Ho [20]. High-resolution optical absorption spectra of an intrinsic $R^{3+}$ ion and $\mathrm{Er}^{3+}$ probe further confirmed the magnetic ordering temperatures and proposed that the magnetic structures of the copper subsystem are same for all the members of $R_{2} \mathrm{BaCuO}_{5}(R=\mathrm{Sm}, \mathrm{Eu}, \mathrm{Tm}, \mathrm{Yb}, \mathrm{Lu})$ [21]. Furthermore, the optical spectra in the "green phase" of $\mathrm{Dy}_{2} \mathrm{BaCuO}_{5}$ proposed the first-order magnetic phase transition involving $\mathrm{Cu}$ and Dy moments [22]. Neutron diffraction studies have been performed for $\mathrm{Nd}$ and $\mathrm{Gd}$ compounds at the $R$ site. The magnetic structure of $\mathrm{Nd}_{2} \mathrm{BaCuO}_{5}$ was suggested on the basis of a propagation vector $k=[0,0,1 / 2]$ below the Néel temperature of $7.8 \mathrm{~K}$, where the $\mathrm{Cu}^{2+}$ and $\mathrm{Nd}^{3+}$ magnetic moments were aligned along the crystallographic $c$ axis and in the $a b$ plane, respectively [23]. Recent neutron results on isotopically substituted ${ }^{160} \mathrm{Gd}_{2} \mathrm{BaCuO}_{5}$ proposed an incommensurate magnetic structure with a propagation vector $k=[0,0,1 / 2-\delta]$ below $12 \mathrm{~K}$, followed by a commensurate structure with a $k=[0,0,1 / 2]$ propagation vector at $5 \mathrm{~K}$, which was accompanied with a Gd spin reorientation and a magnetostructural transition [24]. The considerable magnetic refrigeration with a maximum magnetic entropy change of 

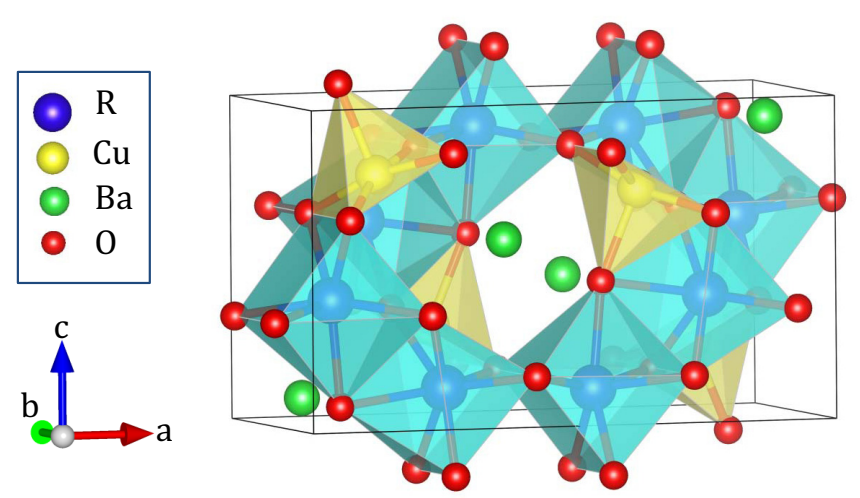

FIG. 1. Direct linkage of $\mathrm{RO}_{7}$ polyhedra and linkage through the $\mathrm{CuO}_{5}$ polyhedra via $\mathrm{O}$ atoms. Atomic positions are described in the figure.

$-\Delta \mathrm{S}_{M} \approx 10.4 \mathrm{~J} \mathrm{~kg}^{-1} \mathrm{~K}^{-1}$ at Néel temperature and refrigerant capacity of $\approx 263 \mathrm{~J} / \mathrm{kg}$ were suggested for $\mathrm{Ho}_{2} \mathrm{BaCuO}_{5}$ [25].

Recently, the spin-chain compounds $R_{2} \mathrm{BaNiO}_{5}$ [26-31] and $R_{2} \mathrm{BaCoO}_{5}$ [32] attract special attention in multiferroics, proposing different origins behind the occurrence of ferroelectric order. In this study, we also observe the ferroelectric order in the unexplored $R_{2} \mathrm{BaCuO}_{5}(R=\mathrm{Er}, \mathrm{Dy}, \mathrm{Sm})$ series. Here, we consider the representative of the rare-earth elements as Er and Dy with the lower ionic radius and Sm as a rare-earth representative with the higher ionic radius. Intriguingly, the ferroelectricity in all the members occurs at much higher temperatures than the magnetic ordering temperatures. The values of ferroelectric $(\mathrm{FE})$ ordering temperatures $\left(T_{\mathrm{FE}}\right)$ are close to room temperature as $\sim 235$ and $\sim 232 \mathrm{~K}$ for $\mathrm{Er}_{2} \mathrm{BaCuO}_{5}$ $(\mathrm{EBCO})$ and $\mathrm{Dy}_{2} \mathrm{BaCuO}_{5}(\mathrm{DBCO})$, respectively, whereas the value is $\sim 184 \mathrm{~K}$ for $\mathrm{Sm}_{2} \mathrm{BaCuO}_{5}(\mathrm{SBCO})$. The values of $P$ are high as $\sim 1410, \sim 992$, and $\sim 980 \mu \mathrm{C} / \mathrm{m}^{2}$ for $\mathrm{E}=4 \mathrm{kV} / \mathrm{cm}$ in the case of $\mathrm{EBCO}, \mathrm{DBCO}$, and $\mathrm{SBCO}$, respectively. An interesting ME coupling driven by the magnetic field cooled (FC) effect is observed below the magnetic ordering temperatures for all the members of the series of compounds. The magnetic FC effect driven increase of $P$ is similar for EBCO and DBCO, which is different for SBCO. These FC effects are strongly correlated to the MD effect as well as the magnetization curves of $R_{2} \mathrm{BaCuO}_{5}$. The synchrotron diffraction studies of DBCO over a wide temperature range of $10-300 \mathrm{~K}$ confirm a structural transition to a polar structure of $P n a 2_{1}$ space group from the centrosymmetric Pnma structure at $232 \mathrm{~K}$, around which the ferroelectricity appears. The results indicate that the ferroelectricity appears due to the structural transition to a polar structure.

\section{EXPERIMENTAL DETAILS}

Polycrystalline $R_{2} \mathrm{BaCuO}_{5}(R=\mathrm{Er}$, Dy, Sm) compounds are prepared using the solid-state reaction [14]. The singlephase chemical composition is confirmed by the x-ray diffraction studies at room temperature recorded in a PANalytical X-ray diffractometer (Model: X' Pert PRO) using the $\mathrm{Cu} K_{\alpha}$ radiation. The single-phase chemical composition is further checked by the synchrotron x-ray diffraction studies recorded with a wavelength of $0.1259 \AA(98 \mathrm{keV})$ at the P07 beamline of PETRA III, Hamburg, Germany in the temperature range of 10-300 K. The synchrotron powder diffraction data are analyzed using the Rietveld refinement with the commercially available MAUD and FULLPROF softwares. Powder sample, pressed into a pellet, is used for the dielectric measurements using an E4980A LCR meter (Agilent Technologies, USA) equipped with a PPMS-II system of Quantum Design. The pyroelectric current $\left(I_{p}\right)$ is recorded in an electrometer (Keithley, model $6517 \mathrm{~B}$ ) at a constant temperature sweep rate. The $I_{p}$ is integrated over time for obtaining the spontaneous electric polarization. The poling electric field is applied during cooling processes and the measurements are carried out in the warming mode with a zero electric field. Before the measurement of $I_{p}$, the electrical connections are short circuited and waited for a long time. In all the measurements, the electrical contacts are fabricated using an air drying silver paint. The temperature dependent heat capacity $\left(C_{p}\right)$ is measured with a PPMS-I system of Quantum Design. Magnetization is measured in a commercial magnetometer of Quantum Design (MPMS, evercool) both in zero-field-cooled (ZFC) and FC protocols. In the case of $\mathrm{ZFC}$ and $\mathrm{FC}$ conditions sample is cooled in zero magnetic field $(H)$ and nonzero $H$ and measurements are carried out in the warming mode with a nonzero $H$.

\section{EXPERIMENTAL RESULTS AND DISCUSSIONS}

Thermal variations of the ZFC-FC magnetization curves recorded at 100 Oe are displayed in Figs. 2(a), 2(b), and 2(c)
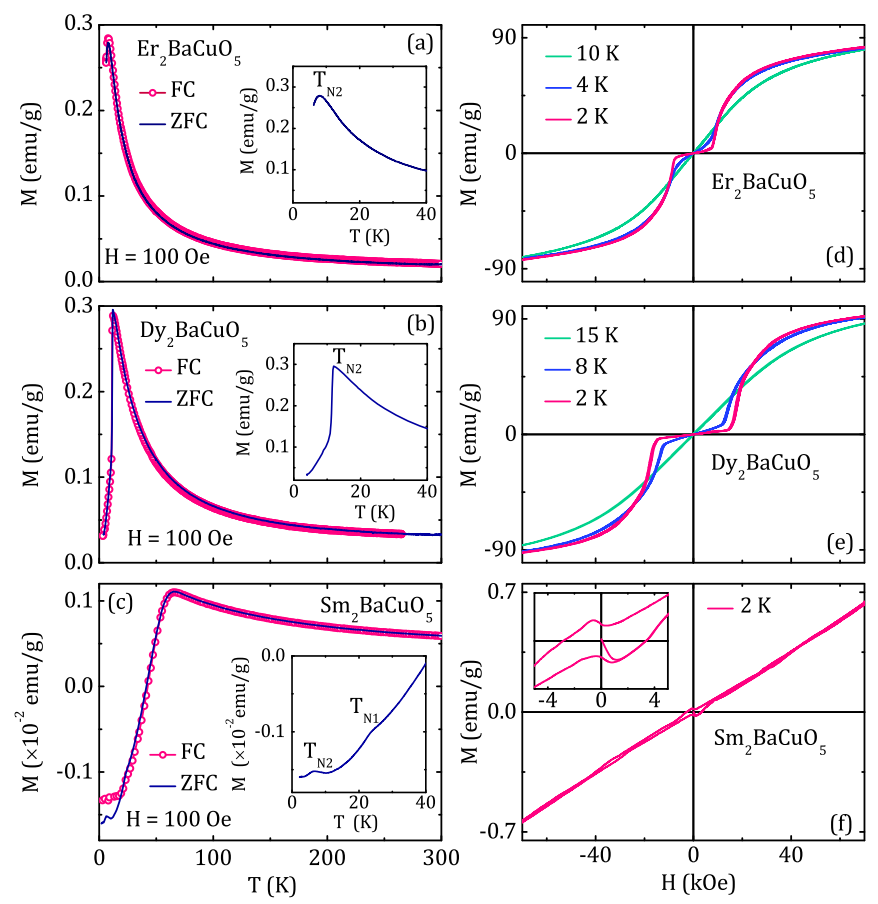

FIG. 2. Temperature dependencies of FC-ZFC magnetization curves for (a) EBCO, (b) DBCO, and (c) SBCO recorded with 100 Oe. The insets highlight the low- $T$ region of each $M(T)$ curve. Magnetization curves for (d) EBCO, (e) DBCO, and (f) SBCO at selected temperatures. Inset of (f) highlights the low-field region of the $M(H)$ curve. 
for EBCO, DBCO, and SBCO, respectively. In accordance with the reported results, a maximum representing the antiferromagnetic (AFM) ordering of the $\mathrm{R}$ ion $\left(T_{N 2}\right)$ is observed at $\sim 5$ and $11 \mathrm{~K}$ for EBCO and DBCO, respectively $[18,19]$. The insets of Figs. 2(a) and 2(b) highlight the low-temperature transitions. A sharp fall of magnetization below $\sim 11 \mathrm{~K}$ rather proposes the first-order magnetic phase transition, as originally suggested from the optical studies in DBCO [22]. Any definite signature of similar first-order magnetic phase transition is missing for EBCO in the current results because of the limitation of the measurement facility below $2 \mathrm{~K}$. The nature of the ZFC-FC magnetization curves for SBCO is quite different from the other two compounds. As shown in Fig. 2(c), the $M(T)$ decreases rapidly below $\sim 100 \mathrm{~K}$ and becomes negative below $50 \mathrm{~K}$. Similar $M(T)$ curves have been noted for the different systems proposing different origins [33]. The interplay between different temperature dependencies of the rare-earth and $3 d$ moments were correlated to the negative magnetization in the $3 d-4 f$ oxide systems. Along with the negative magnetization, a weak magnetization is noted in SBCO than the other two compounds. This might be attributed to the close ordered moments of $\mathrm{Sm}^{3+}$ and $\mathrm{Cu}^{2+}$ ions with an antiparallel exchange coupling. As depicted in the inset of Fig. 2(c), two anomalies are observed around $\sim 23 \mathrm{~K}\left(T_{N 1}\right)$ and $6 \mathrm{~K}\left(T_{N 2}\right)$, which represent the AFM ordering temperatures of $\mathrm{Cu}^{2+}$ and $\mathrm{Sm}^{3+}$, respectively $[18,19]$. We further note that the ordered moments of $\mathrm{Er}^{3+}$ and $\mathrm{Dy}^{3+}$ are much larger than the $\mathrm{Cu}^{2+}$ moment, which primarily distinguishes EBCO and DBCO from SBCO and leads to the different magnetization results.

The magnetic hysteresis loops are recorded below the transition temperatures as depicted in Figs. 2(d), 2(e), and 2(f) for EBCO, DBCO, and SBCO, respectively. The EBCO and DBCO show field induced transition from AFM to a ferromagnetic (FM) state below $T_{N 2}$. The field induced transition shifts toward the lower magnetic field with increasing temperature and vanishes above $T_{N 2}$. The nature of $M(H)$ curve is different for SBCO. An almost linear magnetization curve is observed at $2 \mathrm{~K}$, as depicted in Fig. 2(f), which is consistent with the proposed AFM order. The nature of the $M(H)$ curve in the low-field region is highlighted in the inset of Fig. 2(f). A negative magnetization is observed in the low positive magnetic field up to $3 \mathrm{kOe}$. This negative magnetization below $3 \mathrm{kOe}$ is consistent with the negative $M(T)$ curve in the low temperature recorded at $100 \mathrm{Oe}$.

To find out the onset of $\mathrm{Cu}^{2+}$ ordering, which is not evident in the $M(T)$ curve of EBCO and DBCO, we incorporate the specific heat capacity $\left(C_{P}\right)$ measurements. Figures 3(a) and 3(b) show the $C_{P}(T)$ of EBCO and DBCO, respectively. The insets of the two figures highlight the low-temperature region of $C_{P}(T)$. A second anomaly $\left(T_{N 1}\right)$ is evident around $\sim 19$ and $\sim 18 \mathrm{~K}$ for EBCO and DBCO, respectively, which is associated with the strong signature of $T_{N 2}$ at low temperature $[16,18]$. In addition to the low-temperature results, the weak anomalies, as indicated by the arrows in Figs. 3(a) and 3(b) for EBCO and DBCO, respectively, are also observed around $\sim 235$ and $\sim 232 \mathrm{~K}$. We note that the onset of the polar order is noted close to those temperatures for EBCO and DBCO, which is discussed elsewhere in this paper.

The dielectric permittivity $(\epsilon)$ is recorded at different frequencies $(f)$ by varying $T$ for all three compounds.
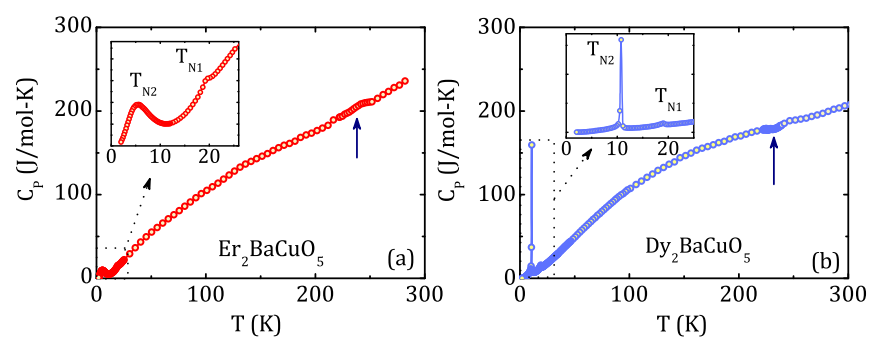

FIG. 3. The $T$ variations of heat capacity $\left(C_{p}\right)$ for (a) EBCO and (b) DBCO. The inset of all figures show the highlighted region of $C_{p}$. The arrows in the high-temperature region depict the FE ordering temperature.

Figures 4(a), 4(b), and 4(c) depict thermal variations of the real components $\left(\epsilon^{\prime}\right)$ of $\epsilon$ for $\mathrm{EBCO}$, DBCO, and SBCO, respectively. The $\epsilon^{\prime}(T)$ plots demonstrate a weak anomaly or a change of slope in the $\epsilon^{\prime}(T)$ curve in the high-temperature region, similar to that observed in the $C_{P}(T)$ curve, which is indicated by the arrows in the figures. Here, the arrows indicate the onset of the spontaneous electric polarization. The details of which are discussed later. A peak or anomaly is also observed in the low- $T$ region, as indicated by the another arrows in the figures, around which the $T_{N 1}$ is observed for all the three compounds, indicating the ME coupling.

The magnetodielectric (MD) effects are recorded at low temperatures for all the compounds. The MD effects, defined as $\epsilon^{\prime}(H) / \epsilon^{\prime}(0)-1$, are depicted with $H$ in Figs. 4(d)-4(f) for EBCO, DBCO, and SBCO. The $\epsilon^{\prime}(H)$ and $\epsilon^{\prime}(0)$ represent the $\epsilon^{\prime}$ with $H$ and $H=0$, respectively. As depicted in Figs. 4(d) and 4(e), the $H$ variations of MD effect for EBCO and DBCO are correlated to the observed magnetization curves at $2 \mathrm{~K}$.
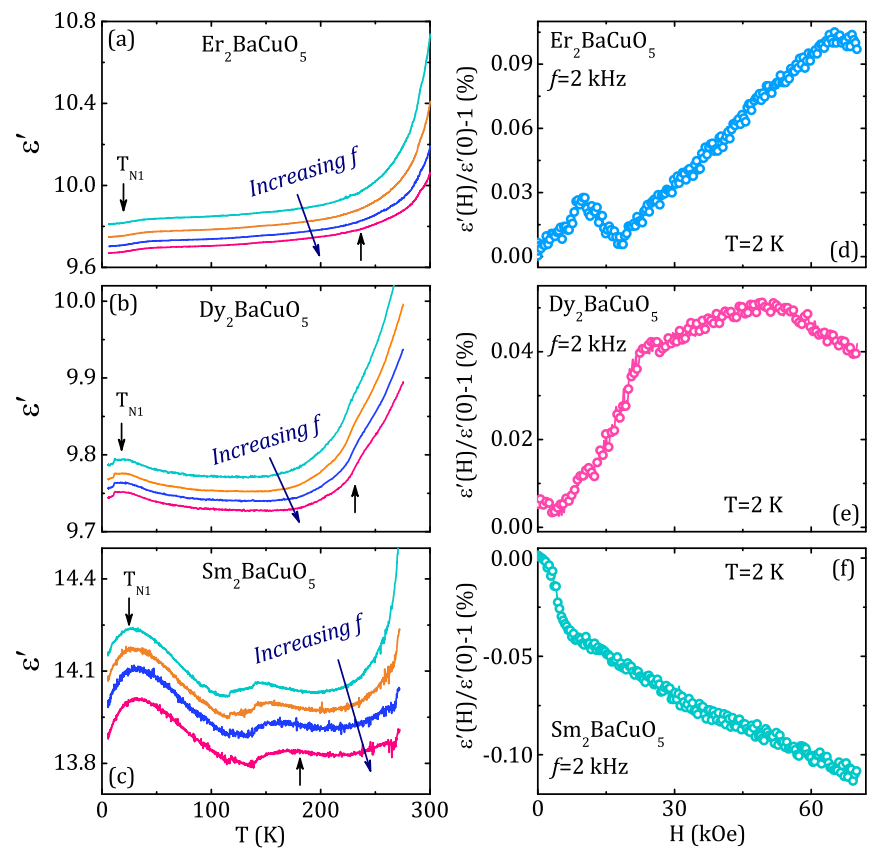

FIG. 4. The $T$ variations of real the component of dielectric permittivities $\left(\epsilon^{\prime}\right)$ at different frequencies $(f)$ for (a) EBCO, (b) DBCO, and (c) SBCO. The arrows in the high-temperature region depict the FE ordering temperature. The magnetodielectric response defined as, $\epsilon^{\prime}(H) / \epsilon^{\prime}(0)-1$, with $H$ for (d) EBCO, (e) DBCO, and (f) SBCO. 

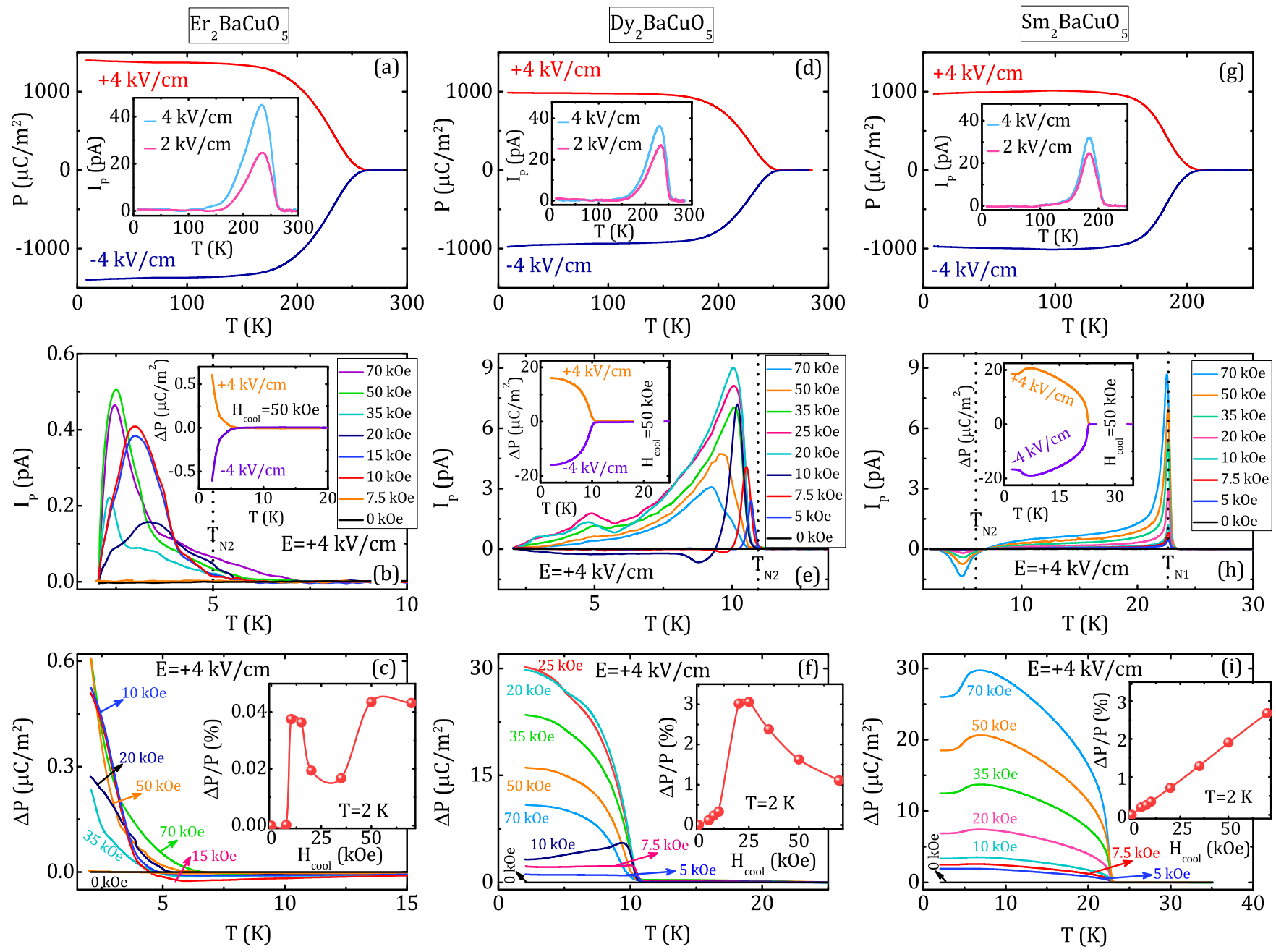

FIG. 5. The $T$ variations of polarization $(P)$ estimated at opposite poling fields $(E= \pm 4 \mathrm{kV} / \mathrm{cm})$ of (a) EBCO, (d) DBCO, and (g) SBCO. Insets show $T$ variations of pyroelectric current $\left(I_{p}\right)$ at two different $E$. The $T$ variations of $I_{p}$ curves for different magnetic cooling fields ( $\left.H_{\text {cool }}\right)$ of (b) EBCO, (e) DBCO, and (h) SBCO. The positions of $T_{N 1}$ and $T_{N 2}$ are given by the vertical broken lines. Insets shows the $\Delta P(T)$ curves with $H_{\text {cool }}=50 \mathrm{kOe}$ and $E= \pm 4 \mathrm{kV} / \mathrm{cm}$. The $T$ variations of $\Delta P(T)$ curves for different $H_{\text {cool }}$ of (c) EBCO, (f) DBCO, and (i) SBCO. Insets of the corresponding figures show the variations of $\Delta P / P$ with $H_{\text {cool }}$.

The changes in the $\mathrm{MD}(\%)$ curve at $2 \mathrm{~K}$ are rapid around $\sim 7.5$ and $\sim 20 \mathrm{kOe}$, around which the $H$ induced transitions are noticed in the magnetization curves at $2 \mathrm{~K}$ for EBCO and DBCO, respectively. With the further increase of $H$ the $\mathrm{MD}(\%)$ effect of EBCO shows a peak around $\sim 8 \mathrm{kOe}$, above which it decreases showing a "dip," and finally it increases systematically above $\sim 18 \mathrm{kOe}$. The $\mathrm{MD}(\%)$ effect of $\mathrm{DBCO}$ is different from the result of EBCO. It shows a change of slope around $\sim 23 \mathrm{kOe}$, above which it further increases displaying a maximum around $\sim 50 \mathrm{kOe}$. The MD effect of SBCO is different from the rest of the two. Initially, it decreases rapidly, around which a negative $M(H)$ with the increase of low $H$ up to $\sim 3 \mathrm{kOe}$ is observed in the magnetization curve at $2 \mathrm{~K}$. With further increase in $H$, the $\mathrm{MD}(\%)$ decreases monotonically at $2 \mathrm{~K}$.

In order to confirm the occurrence of spontaneous polar order, the pyroelectric currents $\left(I_{p}\right)$ are recorded with $T$ for all the samples in different conditions. A peak in $I_{p}(T)$ is observed for all the samples, as evident in the insets of Figs. 5(a), $5(\mathrm{~d})$, and $5(\mathrm{~g})$ with poling fields $(E)$ of 2 and $4 \mathrm{kV} / \mathrm{cm}$ for
EBCO, DBCO, and SBCO, respectively. The peaks of $I_{p}(T)$ curves appear at 235, 232, and $184 \mathrm{~K}$ for $\mathrm{EBCO}, \mathrm{DBCO}$, and SBCO, respectively. To confirm the peaks in $I_{p}(T)$, appear due to genuine occurrence of the polar order, the $I_{p}(T)$ are recorded for different poling temperatures, which are above and below the peak-temperatures for all three compounds. In all the measurements the definite signatures of the peaks are always observed at the same temperature, as evident in Fig. S1 of Ref. [34], pointing genuine occurrence of the polar order at the peaks in $I_{p}(T)$ curves for all three materials. The integral of $I_{p}(T)$ over time gives the reproducible value of $P(T)$. The polarization $(P)$ with $T$ for opposite $E$ are depicted in Figs. 5(a), 5(d), and 5(g) for EBCO, DBCO, and SBCO, respectively. The reversal of $P(T)$ due to a change in sign of $E( \pm 4 \mathrm{kV} / \mathrm{cm})$ signifies the ferroelectric behavior of the compounds. Importantly, the values of $P$ in the current investigation are $\sim 1410, \sim 992$, and $\sim 980 \mu \mathrm{C} / \mathrm{m}^{2}$ for EBCO, DBCO, and SBCO, respectively for $E=4 \mathrm{kV} / \mathrm{cm}$. The $P$ values of $\mathrm{EBCO}$ and $\mathrm{DBCO}$ are $\sim 10$ and $\sim 245$ times higher than the reported values of $P$ for $\mathrm{Er}_{2} \mathrm{BaNiO}_{5}$ and $\mathrm{Dy}_{2} \mathrm{BaNiO}_{5}$ 
[30,35], whereas the $P$ value of $\mathrm{Sm}_{2} \mathrm{BaCuO}_{5}$ is quite close the reported $P$ value of $\mathrm{Sm}_{2} \mathrm{BaNiO}_{5}$ [26].

In order to investigate the possible ME coupling, the $I_{p}(T)$ curves are recorded in different conditions of magnetic FC effects after cooling from $T>T_{N 1}$ or $T_{N 2}$, separately. The magnetic FC effects are applied with three different conditions: (1) ZFC, (2) FC protocols as described in the experimental section, and (3) cooling in a nonzero $H$ and measurements of $I_{p}(T)$ in the warming mode in zero magnetic field. In the above three cases the magnetic field cooling temperature and electric field poling temperature are considered the same. Intriguingly, a definite signature of the peak in the $I_{p}(T)$ curve, pointing additional change in $P(\Delta P)$, is always noted for case 2 , i.e., in the typical magnetic FC protocol only. In the other two cases the peak in the $I_{p}(T)$ curves is always absent for the three samples. In addition, during the record of $I_{p}(T)$ curve with FC protocol as described in case 2, the cooling from $T>T_{N 1}$ as well as $T_{N 2}$ provides the same result for EBCO and $\mathrm{DBCO}$. This confirms that the $\mathrm{R}$ ordering at $T_{N 2}$ is the key for the occurrence of magnetic $\mathrm{FC}$ driven $\Delta P$, whereas $\mathrm{Cu}$ ordering at $T_{N 1}$ does not influence on the occurrence of ferroelectricity. The detailed results of the $I_{p}(T)$ curves for different magnetic field cooling processes are described in Fig. S2 of Ref. [34] for DBCO.

The results on the occurrence of $\Delta P$ due to the magnetic FC effect are depicted in Figs. 5(b) and 5(c) for EBCO, Figs. 5(e) and 5(f) for DBCO, and Figs. 5(h) and 5(i) for SBCO. In Fig. 5(b) the $I_{p}(T)$ curves are depicted for different cooling fields $\left(H_{\text {cool }}\right)$. The peaks in the $I_{p}(T)$ curves are observed below $T_{N 2}$ of EBCO and are strongly influenced by $H_{\text {cool }}$. The peak observed in the $I_{p}(T)$ curves shifts with $H_{\text {cool }}$ and the magnitude is strongly dependent on $H_{\text {cool }}$. The $\Delta P(T)$ curves, as obtained from the time integration of $I_{p}(T)$ curves for $E= \pm 4 \mathrm{kV} / \mathrm{cm}$ and $H_{\text {cool }}=50 \mathrm{kOe}$, are depicted in the inset of Fig. 5(b). The reversal of $\Delta P$ due to opposite poling field ensures the ferroelectric nature of the magnetic FC driven occurrence of $\Delta P$. The $\Delta P$ at different $H_{\text {cool }}$ are depicted in Fig. 5(c), where inset of the figure shows the plot of $\Delta P / P$ with $H_{\text {cool }}$. Here, the $\Delta P$ and $P$ at $2 \mathrm{~K}$ are the change in polarization driven by the FC effect and the value of the electric polarization recorded in zero magnetic field for EBCO, as depicted in Fig. 5(a). The plot of $\Delta P / P$ with $H_{\text {cool }}$ at $2 \mathrm{~K}$ exhibits similar $H$ dependence of MD for EBCO. As observed in the MD with $H$ plot, the $\Delta P / P$ plot shows a sharp rise above $7.5 \mathrm{kOe}$ and is followed by a decrease displaying a dip, above which it increases with increasing $H_{\text {cool }}$. The results of $I_{p}(T)$ and $\Delta P(T)$ curves for DBCO are depicted in Figs. 5(e) and 5(f), respectively. The peaks observed in the $I_{p}(T)$ curve are found just below $T_{N 2}$. Analogous to the results of EBCO, peak in the $I_{p}(T)$ curves shifts and the value of $\Delta P$ changes significantly depending on $H_{\text {cool }}$. As depicted in the inset of Fig. 5(f), the $\Delta P / P$ with $H_{\text {cool }}$ at $2 \mathrm{~K}$ exhibits similar behavior of the MD vs $H$ plot for DBCO. A sharp increase in $\triangle P / P$ is observed close to $\sim 20 \mathrm{kOe}$, analogous to that observed magnetic field driven transition at $20 \mathrm{kOe}$ in the magnetization curve at $2 \mathrm{~K}$. The $\Delta P / P$ shows a decrease with further increase in $H_{\text {cool }}$. The results of SBCO are different from the results of EBCO and DBCO. A sharp peak in the $I_{p}(T)$ curve is observed at $T_{N 1}$, which is missing for EBCO and DBCO. In addition, another
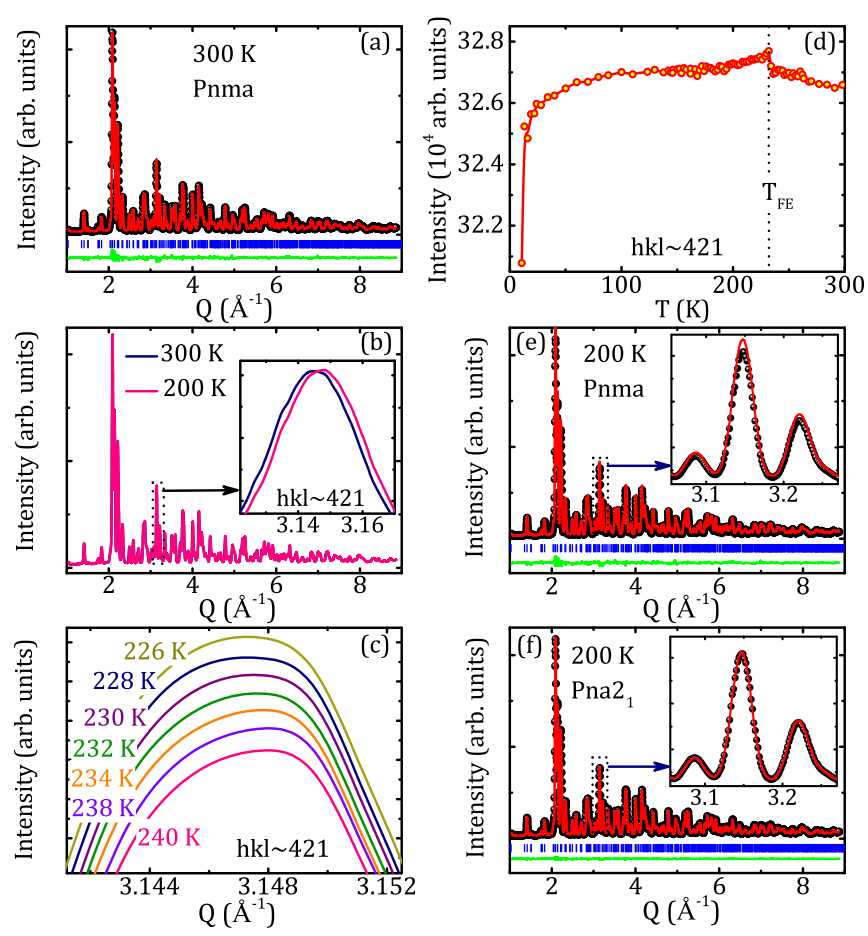

FIG. 6. (a) The Rietveld refinement of the synchrotron powder diffraction pattern (symbols) at $300 \mathrm{~K}$ for DBCO. The solid curve demonstrates the fit. (b) Synchrotron diffraction patterns at 200 and $300 \mathrm{~K}$. Inset highlights the (421) peak. (c) The (421) peak positions with $T$ in a small interval between $226-240 \mathrm{~K}$. (d) $T$ variation of the integrated intensity of (421) peak. Rietveld refinements of the diffraction patterns at $200 \mathrm{~K}$ using (e) Pnma and (f) $P n a 2_{1}$ space groups. Insets further highlight the quality of the refinements in a small $Q$ region.

peak in the $I_{p}(T)$ curve is observed, which is opposite in direction below $T_{N 2}$. Both the peaks in the $I_{p}$ curve remain at the same position for all the values of $H_{\text {cool }}$. The plot of $\Delta P / P$ with $H_{\text {cool }}$ shows nearly linear dependence at $2 \mathrm{~K}$, as also observed in the magnetization curve as well as MD vs $H$ plot in the high-field region for SBCO.

To find out the origin of ferroelectricity at much higher temperatures than the magnetic ordering temperatures, the structural properties are investigated by the synchrotron diffraction studies over a temperature range of $10-300 \mathrm{~K}$ for $\mathrm{DBCO}$, as a representative of isostructural $R_{2} \mathrm{BaCuO}_{5}$ series. An example of a diffraction pattern together with the refinement with a Pnma space group at $300 \mathrm{~K}$ is shown in Fig. 6(a). The blue colored bars below the diffraction pattern represent the diffraction peak positions. The green colored curve at the bottom show the difference plot and confirms absence of the impurity phases. The lattice parameters, atomic positions, thermal displacement parameters, and reliability parameters at $300 \mathrm{~K}$ are given in Table I . Figure 6(b) shows the diffraction patterns of DBCO above $T_{\mathrm{FE}}$ at $300 \mathrm{~K}$ and below $T_{\mathrm{FE}}$ at $200 \mathrm{~K}$ together in the same plot. The inset of Fig. 6(b) highlights the change in intensity pattern of the (421) diffraction peak associated with a shift of the peak position. The changes in the (421) diffraction peak position are depicted in Fig. 6(c) from 240 to $226 \mathrm{~K}$ with the small temperature intervals. Here, the (421) peak at different temperatures is 
TABLE I. Lattice parameters, atomic positions, thermal displacement parameters, and reliability parameters of DBCO at selected temperatures. $\mathrm{B}^{*}$ stands for the thermal displacement parameter.

\begin{tabular}{|c|c|c|c|c|c|c|}
\hline$T(\mathrm{~K})$ & Space group & Lattice parameters $(\AA)$ & Atoms & $x, y, z$ & $\mathrm{~B}^{*}$ & Reliability parameters \\
\hline 300 & Pnma & $\begin{array}{c}a=12.2243(9) \\
b=5.6808(4) \\
c=7.1539(4)\end{array}$ & $\begin{array}{l}\mathrm{Ba} \\
\mathrm{Dy} 1 \\
\mathrm{Dy} 2 \\
\mathrm{Cu} \\
\mathrm{O} 1 \\
\mathrm{O} 2 \\
\mathrm{O} 3\end{array}$ & $\begin{array}{c}0.9051(4), 0.25,0.9295(6) \\
0.2892(8), 0.25,0.1160(5) \\
0.0738(9), 0.25,0.3969(4) \\
0.6596(3), 0.25,0.7166(1) \\
0.4278(4), 0.0043(5), 0.1555(1) \\
0.2275(2), 0.5074(0), 0.3534(4) \\
0.0905(4), 0.25,0.0668(9)\end{array}$ & $\begin{array}{l}0.63(2) \\
0.30(6) \\
0.17(7) \\
0.58(2) \\
0.48(5) \\
0.76(3) \\
0.39(1)\end{array}$ & $\begin{array}{c}R_{W}(\%)=2.30 \\
R_{\exp }(\%)=1.92 \\
\sigma=0.023\end{array}$ \\
\hline 200 & $P n a 2_{1}$ & $\begin{array}{c}a=12.2192(2) \\
b=7.1492(2) \\
c=5.6783(6)\end{array}$ & $\begin{array}{l}\mathrm{Ba} \\
\mathrm{Dy} 1 \\
\mathrm{Dy} 2 \\
\mathrm{Cu} \\
\mathrm{O} 1^{1} \\
\mathrm{O} 1^{2} \\
\mathrm{O} 2^{1} \\
\mathrm{O} 2^{2} \\
\mathrm{O} 3\end{array}$ & $\begin{array}{l}0.9057(4), 0.9293(7), 0.7573(2) \\
0.2894(3), 0.1168(2), 0.7536(9) \\
0.0741(1), 0.3980(7), 0.7559(3) \\
0.6586(7), 0.7159(1), 0.7812(1) \\
0.4172(5), 0.1608(1), 0.0002(1) \\
0.9458(4), 0.3470(3), 0.4943(5) \\
0.2200(9), 0.3542(1), 0.5413(1) \\
0.7301(1), 0.1612(5), 0.0480(8) \\
0.0913(7), 0.0687(1), 0.7991(2)\end{array}$ & $\begin{array}{l}0.63(3) \\
0.31(5) \\
0.16(7) \\
0.57(8) \\
0.49(1) \\
0.47(6) \\
0.76(7) \\
0.75(5) \\
0.38(7)\end{array}$ & $\begin{array}{c}R_{W}(\%)=2.15 \\
R_{\exp }(\%)=1.91 \\
\sigma=0.028\end{array}$ \\
\hline
\end{tabular}

vertically shifted for the clarification of the small changes in the peak position. The temperature variation of the integrated intensity of the (421) diffraction peak is depicted in Fig. 6(d), which displays an evident signature near $T_{\mathrm{FE}}$. This signature around $T_{\mathrm{FE}}$ in the integrated intensity plot is correlated with the change in the scattering cross section and may point to a possible structural transition. The change in the integrated intensity is similar to that observed for the reported ferroelectric materials, where ferroelectricity appeared due to structural transition [26,36-38]. With further decreasing temperature a sharp fall is observed around $T_{N 1}$, as depicted in Fig. 6(d), which indicates a strong signature of the magnetoelastic coupling. The possible occurrence of the magnetoelastic coupling at $T_{N 2}$ is beyond the scope of our synchrotron data, which is recorded up to $10 \mathrm{~K}$.

The diffraction patterns are refined with the hightemperature Pnma space group in the entire recorded temperature range. We note that the refinement is not satisfactory below $T_{\mathrm{FE}}$ using the Pnma space group, as depicted in Fig. 6(e). Thus a structural transformation from Pnma to a polar structure is proposed for justifying the occurrence of a polar order. We use AMPLIMODE [39] and ISODISTORT [40] software to find out possible noncentrosymmetric space groups having a polar structure below $T_{\mathrm{FE}}$. Among the all possible noncentrosymmetric structures the $P n a 2_{1}$ space group has the highest symmetry, which is also a polar structure. The best fit is realized for the $P n a 2_{1}$ space group. An example of a satisfactory fit with the $P n a 2_{1}$ space group at $200 \mathrm{~K}$ is shown in Fig. 6(f). Insets of the Figs. 6(e) and 6(f) clearly demonstrate the refinements in a small $Q$ range and authenticate the better fit of the diffraction pattern using $P n a 2_{1}$ than the Pnma space group. The refined lattice parameters, atomic positions, thermal displacement parameters, and reliability parameters at $200 \mathrm{~K}$ are listed in Table I. The blue colored bars below the diffraction patterns represent the diffraction peak positions. The difference plot is shown by the green color at the bottom and confirms absence of the impurity peaks.
Thermal variations of the lattice parameters, $a, b$, and $c$, as obtained from the refinements, are depicted in Figs. 7(a), 7(b), and 7(c), respectively. The $T_{\mathrm{FE}}$ is shown by the vertical broken line in the figures. As depicted in Figs. 7(b), and 7(c), the $b$ and $c$ axes in Pnma become $c$ and $b$ axes in $P n a 2_{1}$ structure. An anomaly around $T_{\mathrm{FE}}$ is observed in $a(T)$ and $b(T)$, which is not so apparent in $c(T)$. Thermal variation of the unit cell volume is depicted in Fig. 7(d), where inset of the figure magnifies the region close to $T_{\mathrm{FE}}$. A small discontinuous change is noted below $T_{\mathrm{FE}}$. The results further indicate that the ferroelectricity in DBCO is correlated to this structural transition from the centrosymmetric Pnma to a polar Pna2 structure. We note that the structural transition involves with the considerable deformations of $\mathrm{CuO}_{5}$ and $\mathrm{DyO}_{7}$ polyhedra. In order to probe these deformations microscopically, the bond lengths and bond angles between different atoms are investigated further.

In the $\mathrm{CuO}_{5}$ pyramids one oxygen (O3) occupies the apex position and out of four basal oxygens two are defined as $\mathrm{O} 1$ and the rest two as $\mathrm{O} 2$ in both the Pnma and Pna2 space groups. The positions of oxygen are shown in the Fig. $8(d)$. The thermal variations of two $\mathrm{Cu}-\mathrm{O} 1\left(d_{\mathrm{Cu}-\mathrm{O} 1}\right)$ and

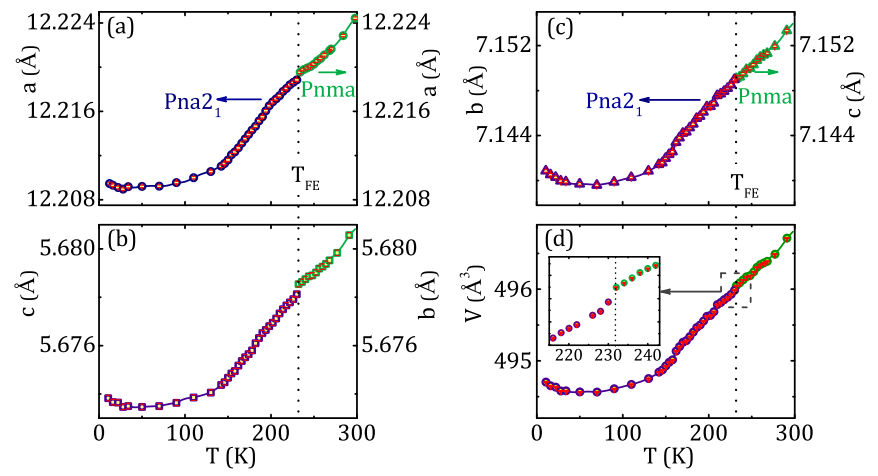

FIG. 7. The $T$ variations of (a) $a$, (b) $b$, (c) $c$, and (d) unit cell volume (V) for DBCO. Inset of (d) magnifies the region close to $T_{\mathrm{FE}}$. The vertical broken line shows the $T_{\mathrm{FE}}$. 

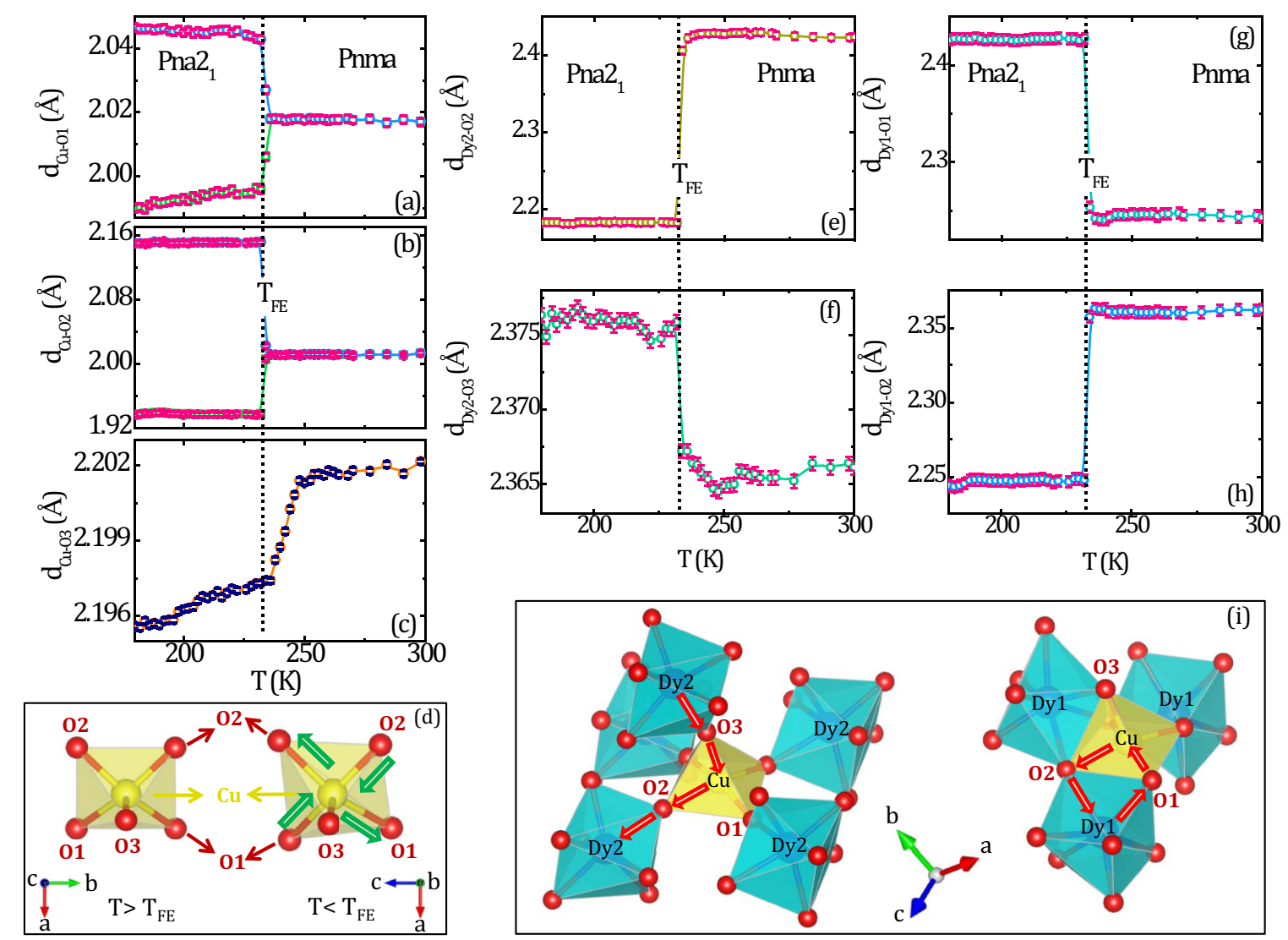

FIG. 8. Variations of (a) Cu-O1 $\left(d_{\mathrm{Cu}-\mathrm{O} 1}\right),\left(\right.$ b) $\mathrm{Cu}-\mathrm{O} 2\left(d_{\mathrm{Cu}-\mathrm{O} 2}\right),(\mathrm{c}) \mathrm{Cu}-\mathrm{O} 3\left(d_{\mathrm{Cu}-\mathrm{O} 3}\right),(\mathrm{e}) \mathrm{Dy} 2-\mathrm{O} 2\left(d_{\mathrm{Dy} 2-\mathrm{O} 2}\right),(\mathrm{f}) \mathrm{Dy} 2-\mathrm{O} 3\left(d_{\mathrm{Dy} 2-\mathrm{O} 3}\right),(\mathrm{g}) \mathrm{Dy} 1-\mathrm{O} 1$ $\left(d_{\text {Dy1-O1 }}\right)$, and (h) Dy1-O2 $\left(d_{\text {Dy1-O2 }}\right)$ bond lengths with $T$ for DBCO. (d) Distortion of the $\mathrm{CuO}_{5}$ pyramid below and above $T_{\mathrm{FE}}$. (i) Distortions of the connecting $\mathrm{DyO}_{7}$ and $\mathrm{CuO}_{5}$ polyhedra below $T_{\mathrm{FE}}$ involving Dy1 (right) and Dy2 (left).

two $\mathrm{Cu}-\mathrm{O} 2\left(d_{\mathrm{Cu}-\mathrm{O} 2}\right)$ bond lengths are depicted in Figs. 8(a) and 8 (b), respectively. The steplike changes are observed in $d_{\mathrm{Cu}-\mathrm{O} 1}$ and $d_{\mathrm{Cu}-\mathrm{O} 2}$ around $T_{\mathrm{FE}}$. The step-like increase and decrease of $\sim 1.2 \%$ and $1.09 \%$ are noted for $d_{\mathrm{Cu}-\mathrm{Ol}}(T)$, whereas the step-like more stronger increase and decrease of $\sim 7.02 \%$ and $3.68 \%$ are noted for $d_{\mathrm{Cu}-\mathrm{O} 2}(T)$. The decrease in $d_{\mathrm{Cu}-\mathrm{O} 3}(T)$ is found to be small as $\sim 0.18 \%$, as depicted in Fig. 8(c). These results indicate that the distortion of the $\mathrm{CuO}_{5}$ pyramid is significant in the basal plane around $T_{\mathrm{FE}}$. The distortions of $\mathrm{CuO}_{5}$ polyhedra for structural change from Pnma to Pna2 $2_{1}$ space group are depicted in the right panel of Fig. 8(d) by the arrows.

As observed in Fig. 1, the $\mathrm{CuO}_{5}$ polyhedra are connected to each other via $\mathrm{DyO}_{7}$ polyhedra, so the changes in $\mathrm{CuO}_{5}$ polyhedra must influence the neighboring $\mathrm{DyO}_{7}$ polyhedra. Two nonequivalent sites of Dy are defined as Dy1 and Dy2 in this paper. The $\mathrm{Dy} 1 \mathrm{O}_{7}$ and $\mathrm{Dy}_{2} \mathrm{O}_{7}$ prisms are joined by a common face and edge into a Dy 1 Dy $2 \mathrm{O}_{11}$ unit. These units linked by the common edges and faces with $\mathrm{CuO}_{5}$ polyhedra form a three-dimensional network. The linkage of $\mathrm{CuO}_{5}$ polyhedra with $\mathrm{Dy} 1 \mathrm{O}_{7}$ and $\mathrm{Dy} 2 \mathrm{O}_{7}$ are shown separately in the right and left panels, respectively, of Fig. 8(i). $\mathrm{A} \mathrm{CuO}_{5}$ pyramid is connected with five $\mathrm{Dy}_{2} \mathrm{O}_{7}$ polyhedra through five oxygen at the edges. The thermal variations of Dy2-O2 $\left(d_{\text {Dy2-02 }}\right)$ and Dy2-O3 $\left(d_{\text {Dy2-O3 }}\right)$ bond lengths are depicted in Figs. 8(e) and 8(f), respectively. In contrast to the decrease in
$d_{\mathrm{Cu}-\mathrm{O} 3}(T)$ [Fig. 8(c)], an increase in $d_{\mathrm{Dy} 2-\mathrm{O} 3}(T)$ is observed around $T_{\mathrm{FE}}$. Similarly, the observed decrease in $d_{\mathrm{Dy} 2-\mathrm{O} 2}(T)$ is also correlated with the increase of connected $d_{\mathrm{Cu}-\mathrm{O} 2}(T)$ [Fig. 8(b)]. Here, the changes of $d_{\text {Dy2-O2 }}$ and $d_{\text {Dy2-O3 }}$ are $\sim 11.5 \%$ and $0.6 \%$, respectively. On the other hand, the three Dy $1 \mathrm{O}_{7}$ polyhedra are connected by the faces with the $\mathrm{CuO}_{5}$ pyramid. The thermal variations of Dy1-O1 $\left(d_{\text {Dy1-O1 }}\right)$ and Dy1-O2 $\left(d_{\text {Dy1-O2 }}\right)$ bond lengths, depicted in Figs. $8(\mathrm{~g})$ and 8(h), also show a similar contrasting nature with the variation of connecting $d_{\mathrm{Cu}-\mathrm{O} 1}(T)$ and $d_{\mathrm{Cu}-\mathrm{O} 2}(T)$ around $T_{\mathrm{FE}}$. Here, the changes of $d_{\mathrm{Dy} 1-\mathrm{O} 1}$ and $d_{\mathrm{Dy} 1-\mathrm{O} 2}$ are $\sim 8.2 \%$ and $4.5 \%$, respectively. The changes in aforesaid bond lengths are schematically represented in Fig. 8(i) by the arrows. These results clearly imply that the deformations of both $\mathrm{Dy} 1 \mathrm{O}_{7}$ and $\mathrm{Dy} 2 \mathrm{O}_{7}$ polyhedra are strongly influenced by the distortion of $\mathrm{CuO}_{5}$ polyhedra around the FE order in DBCO. The results clearly infer that the emergence of electric polarization involves large distortions of the $\mathrm{CuO}_{5}$ and $\mathrm{DyO}_{7}$ polyhedra. We carefully note that the distortions are maximum for $d_{\mathrm{Dy} 2-\mathrm{O} 2}, d_{\mathrm{Cu}-\mathrm{O} 2}$, and $d_{\text {Dy1-O1 }}$, which are significant. Here, all these bond lengths are directed along (101), suggesting the possible direction of polarization.

The thermal variations of bond angles between $\mathrm{Cu}$ and three $\mathrm{O}$ atoms are depicted in Figs. 9(a-d). As depicted in Figs. 9(a) and 9(b), a sharp decrease of $\sim 3.03 \%$ for $\mathrm{O} 1-\mathrm{Cu}-\mathrm{O} 1$ bond angle and the small increase of $\sim 0.19 \%$ for 

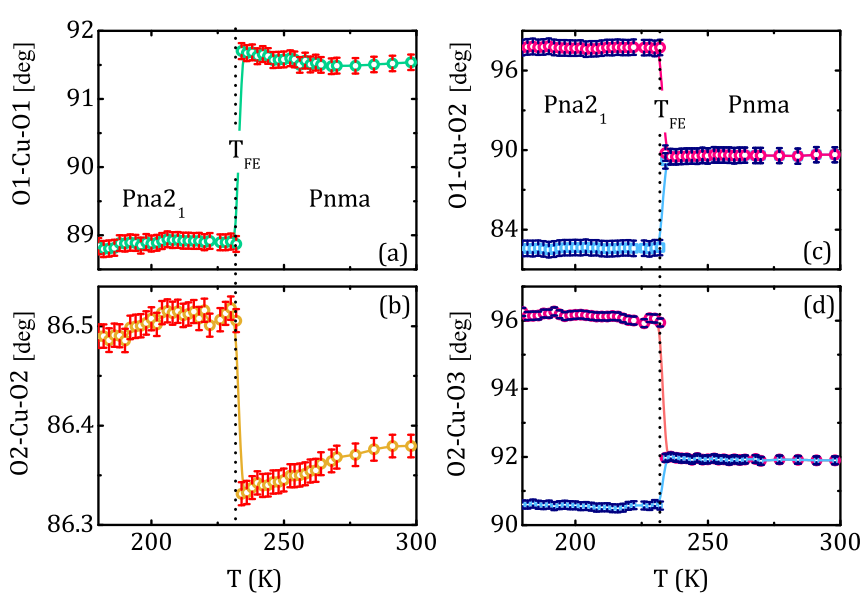

FIG. 9. Variations of (a) O1-Cu-O1, (b) O2-Cu-O2, (c) O1-Cu$\mathrm{O} 2$, and (d) $\mathrm{O} 2-\mathrm{Cu}-\mathrm{O} 3$ bond angles with $T$ for DBCO.

$\mathrm{O} 2-\mathrm{Cu}-\mathrm{O} 2$ bond angle are observed around $T_{\mathrm{FE}}$. The decrease and increase are observed in $\mathrm{O} 1-\mathrm{Cu}-\mathrm{O} 2$ and $\mathrm{O} 2-\mathrm{Cu}-\mathrm{O} 3$ bond angles, respectively, around $T_{\mathrm{FE}}$ in $\mathrm{DBCO}$. The maximum values of changes in $\mathrm{O} 1-\mathrm{Cu}-\mathrm{O} 2$ and $\mathrm{O} 2-\mathrm{Cu}-\mathrm{O} 3$ below $T_{\mathrm{FE}}$ are remarkable as $\sim 9 \%$ (increase), $\sim 8 \%$ (decrease), and $\sim 4.2 \%$ (increase), and $\sim 1.5 \%$ (decrease), respectively. Our micro-structural studies indicate that the distortions of $\mathrm{CuO}_{5}$ polyhedra involve the rotations, which lead to a key role for the ferroelectric order. This is analogous to that observed for $\mathrm{RCrO}_{3}$ [41,42] and $\mathrm{RCrO}_{4}$ [36].

The structural studies propose that the ferroelctricity involves the structural transition at high temperature, which is much above the magnetic order. Thus the influence of the magnetic exchange interaction on the occurrence of FE order is likely to be negligible. A classic Jahn-Teller mechanism is also ruled out due to the absence of the second-order Jahn-Teller active $\left(d^{0}\right.$ and $\left.6 s^{2}\right)$ cations, that often leads to the ferroelectric distortions through the electronic mechanisms. Recently, similar results of the higher $T_{\mathrm{FE}}$ than the magnetic ordering temperature have been observed in few members of the spin-chain compounds $R_{2} \mathrm{BaNiO}_{5}$, such as, $\mathrm{Er}_{2} \mathrm{BaNiO}_{5}$ [35] and $\mathrm{Sm}_{2} \mathrm{BaNiO}_{5}$ [26]. For $\mathrm{Er}_{2} \mathrm{BaNiO}_{5}$ a short-range order driven ferroelectricity was proposed, whereas a structural transition to a noncentrosymmetric polar structure has been proposed for the occurrence of ferroelectricity in the case of $\mathrm{Sm}_{2} \mathrm{BaNiO}_{5}$. Similar results with a much higher $T_{\mathrm{FE}}$ than the magnetic order was observed for $R \mathrm{CrO}_{4}(R=\mathrm{Sm}, \mathrm{Gd}$, and Ho) series, which was proposed to be correlated with the structural transition to a nonpolar structure [36]. Herein, the structural transition to a polar structure of $P n a 2_{1}$ space group is proposed at $T_{\mathrm{FE}}$ from the noncentrosymmetric Pnma space group. This is analogous to that proposed for $R \mathrm{CrO}_{3}$ $(R=\mathrm{Sm}$ and Ho), where the similar structural transition to a polar $P n a 2_{1}$ space group from the Pnma structure was correlated to the FE order with $T_{\mathrm{FE}}>T_{N}$ [41,42]. Recently, the hybrid improper ferroelectricity has been proposed from the calculation for $\mathrm{Ca}_{3} \mathrm{Mn}_{2} \mathrm{O}_{7}$, where the rotational or the tilting distortions generated the electric polarization without the involvement of the polar structure $[43,44]$. This theoretical model has been corroborated by verifying the ferroelectricity from the direct observation for $\mathrm{Ca}_{3} \mathrm{Mn}_{2} \mathrm{O}_{7}$ [45] as well as
$(\mathrm{Ca}, \mathrm{Sr})_{3} \mathrm{SnO}_{7}$ [46]. Consistent with this model, the current investigation shows the similar rotations of the $\mathrm{CuO}_{5}$ polyhedra at $T_{\mathrm{FE}}$, that may lead to the engineering of the polar order in DBCO.

Furthermore, the structural distortion has been suggested for tuning $P$ value in several occasions of films [47-52] as well as polycrystalline compounds [36,53-55]. The influence of structural distortion for the large polarization in bulk perovskite systems was also addressed from the first principles calculations [53]. The structural distortion driven increase of $P$ has been proposed for $A \mathrm{Bi}_{2} \mathrm{Ta}_{2} \mathrm{O}_{9}$ series [54], $\mathrm{Ca}_{1-x} \mathrm{La}_{x} \mathrm{BaCo}_{4} \mathrm{O}_{7}(x \leqslant 0.05)$ [55], and $R \mathrm{CrO}_{4}$ series.[36] Herein, we note the $P$ value is systematic with the ionic radius of $R^{3+}$ in $R_{2} \mathrm{BaCuO}_{5}$, where low ionic radius involves a high value of $P$ for EBCO. With the increase in ionic radius, the $P$ value decreases drastically for $\mathrm{DBCO}$ and it continues to decrease slowly for SBCO. The lower ionic radius of $\mathrm{Er}^{3+}$ might be correlated to the larger structural distortion and thus provides the larger $P$ value for EBCO. In order to establish it, further investigations are suggested on the structural properties of the other members of the $R_{2} \mathrm{BaCuO}_{5}$ series below $T_{\mathrm{FE}}$.

The magnetic FC effect driven occurrence of $\Delta P$ is an another interesting result in the current investigation. In fact, the $\Delta P$ reverses its sign due to change in sign of poling field and proposes the ferroelectric nature of the appearance of $\Delta P$. Here, we emphasize on the fact that an additional increase of polarization occurs only in the case of the magnetic FC effect, which is absent for the $\mathrm{ZFC}$ effect. The contrast results are observed for SBCO compared to the results for the rest two members, where the $\Delta P$ occurs below the $R^{3+}$ ordering for EBCO and DBCO and below the $\mathrm{Cu}^{2+}$ ordering temperature for SBCO. The results may be correlated to the important observations, where the moments of $\mathrm{Sm}^{3+}$ and $\mathrm{Cu}^{2+}$ ions are comparable for SBCO and considerably larger moments of $\mathrm{Er}^{3+}$ and $\mathrm{Dy}^{3+}$ ions are noticed than the $\mathrm{Cu}^{2+}$ moment for $\mathrm{EBCO}$ and $\mathrm{DBCO}$. The $3 d-4 f$ hybridization holds the key for the contrast results. After the magnetic FC process, the possible modification of the magnetic structure, as determined by the neutron diffraction studies, can elucidate on the occurrence of $\Delta P$, which is ferroelectric in nature for $R_{2} \mathrm{BaCuO}_{5}$.

To summarize, the results of the $R_{2} \mathrm{BaCuO}_{5}(R=\mathrm{Er}$, Dy, Sm) series reveal that few members of the $R_{2} \mathrm{BaCuO}_{5}$ series belong to the elite multiferroics family. Members of this series are attractive for the high values of the FE ordering temperature, which varies from 184 to $235 \mathrm{~K}$. The values of the electric polarization are significantly high and belong to the range of $980-1410 \mu \mathrm{C} / \mathrm{m}^{2}$ for a $4 \mathrm{kV} / \mathrm{cm}$ poling electric field. Importantly, these values are comparable to the polarization values of the promising multiferroics. Unlike the common occurrence of the ferroelectric order correlated to the magnetic order, the ferroelectricity in the current observation involves a structural transition to a polar structure of the $P n a 2_{1}$ space group. The unusual magnetoelectric consequences in all three members of the $R_{2} \mathrm{BaCuO}_{5}$ family are unique and distinguishes this series from the typical multiferroics. Intriguingly, an additional increase of polarization, which is ferroelectric in nature, appears only for the magnetic field-cooled effect and attracts the community for the fundamental interest. 


\section{ACKNOWLEDGMENTS}

S.G. acknowledges SERB, India project (Project No. SB/S2/CMP-029/2014) for the financial support. S.G. also acknowledges DST, India for the financial support to perform experiment at PETRA III, DESY, Germany for synchrotron diffraction studies (Proposal No. I-20150193).
[1] N. A. Spaldin, S.-W. Cheong, and R. Ramesh, Phys. Today 63(10), 38 (2010).

[2] M. Fiebig, J. Phys. D: Appl. Phys. 38, 123 (2005).

[3] S. W. Cheong and M. Mostovoy, Nat. Mater. 6, 13 (2007).

[4] J. F. Scott, J. Mater. Chem. 22, 4567 (2012).

[5] N. A. Spaldin and M. Fiebig, Science 309, 391 (2005).

[6] R. Ramesh, Nature (London) 461, 1218 (2009).

[7] J. Wang et al., Science 299, 1719 (2003).

[8] R. O. Cherifi, V. Ivanovskaya, L. C. Phillips, A. Zobelli, I. C. Infante, E. Jacquet, V. Garcia, S. Fusil, P. R. Briddon, N. Guiblin, A. Mougin, A. A. Ünal, F. Kronast, S. Valencia, B. Dkhil, A. Barthélémy, and M. Bibes, Nat. Mater. 13, 345 (2014).

[9] T. Kimura, Y. Sekio, H. Nakamura, T. Siegrist, and A. P. Ramirez, Nat. Mater. 7, 291 (2008).

[10] M. Soda, T. Ishikura, H. Nakamura, Y. Wakabayashi, and T. Kimura, Phys. Rev. Lett. 106, 087201 (2011).

[11] C. Michel and B. Raveau, J. Solid State Chem. 43, 73 (1982).

[12] S. F. Whatkins, F. R. Fronczek, K. S. Wheelock, R. G. Goodrich, W. D. Hamilton, and W. W. Johnson, Acta Crystallogr. C 44, 3 (1988).

[13] B. A. Hunter, S. L. Town, R. L. Davis, G. J. Russell, and K. N. R. Taylor, Physica C (Amsterdam) 161, 594 (1989).

[14] A. Salinas-Sanchez, J. L. Garcia-Munoz, J. Rodriguez-Carvajal, R. Saez-Puche, and J. L. Martinez, J. Solid State Chem. 100, 201 (1992).

[15] H. C. Gupta and V. Sharma, J. Raman Spectrosc. 36, 83 (2005).

[16] V. V. Moshchalkov, N. A. Samarin, I. O. Grishchenko, B. V. Mill, and J. Zoubkova, Solid State Commun. 78, 879 (1991).

[17] A. Salinas-Sanchez, R. Sáez-Puche, and M. A. Alario-Franco, J. Solid State Chem. 89, 361 (1990).

[18] R. Z. Levitin, R. V. Mill, V. V. Moshchalkov, N. A. Samarin, V. V. Snegirev, and J. Zoubkova, J. Magn. Magn. Mater. 90-91, 536 (1990).

[19] Yu. A. Koksharov, V. V. Moshalkov, A. A. Gippius, B. V. Mill, Y. Zoubkova, S. V. Gudenko, and A. N. Mezhuev, Physica C (Amsterdam) 185, 1151 (1991).

[20] M. Baran, H. Szymczak, S. A. Klimin, M. N. Popova, R. Z. Levitin, and B. V. Mill', J. Exp. Theor. Phys. 84, 175 (1997).

[21] I. V. Paukov, M. N. Popova, and B. V. Mill, Phys. Lett. A 169, 301 (1992).

[22] M. N. Popova and G. G. Chepurko, JETP Lett. 52, 562 (1990).

[23] R. Sáez-Puche, E. Climent, E. Jiménez-Melero, J. Romero de Paz, J. L. Martínez, and M. T. Fernández-Díaz, J. Alloys Compds. 408, 613 (2006).

[24] A. K. Ovsyanikov, I. V. Golosovsky, I. A. Zobkalo, and I. Mirebeau, J. Magn. Magn. Mater. 353, 71 (2014).

[25] Y. Zhang, H. Li, J. Wang, X. Li, Z. Ren, and G. Wilde, Ceram. Int. 44, 1991 (2018).

[26] A. Indra, K. Dey, S. Majumdar, I. Sarkar, S. Francoual, R. P. Giri, N. Khan, P. Mandal, and S. Giri, Phys. Rev. B 95, 094402 (2017).
[27] S. Sharma, T. Basu, A. Shahee, K. Singh, N. P. Lalla, and E. V. Sampathkumaran, Phys. Rev. B 90, 144426 (2014).

[28] T. Basu, P. L. Paulose, K. K. Iyer, K. Singh, N. Mohapatra, S. Chowki, B. Gonde, and E. V. Sampathkumaran, J. Phys.: Condens. Matter 26, 172202 (2014).

[29] T. Basu, N. Mohapatra, K. Singh, and E. V. Sampathkumaran, AIP Adv. 5, 037128 (2015).

[30] K. Singh, T. Basu, S. Chowki, N. Mahapotra, K. K. Iyer, P. L. Paulose, and E. V. Sampathkumaran, Phys. Rev. B 88, 094438 (2013).

[31] G. Nénert and T. T. M. Palstra, Phys. Rev. B 76, 024415 (2007).

[32] S. K. Upadhyay and E. V. Sampathkumaran, Appl. Phys. Lett. 112, 262902 (2018).

[33] A. Kumar and S. M. Yusuf, Phys. Rep. 556, 1 (2015).

[34] See Supplemental Material at http://link.aps.org/supplemental/ 10.1103/PhysRevB.100.014413 for details of the pyroelectric measurements in different conditions.

[35] T. Basu, V. V. Ravi Kishore, S. Gohil, K. Singh, N. Mohapatra, S. Bhattacharjee, B. Gonde, N. P. Lalla, P. Mahadevan, S. Ghosh, and E. V. Sampathkumaran, Sci. Rep. 4, 5636 (2014).

[36] A. Indra, K. Dey, J. K. Dey, S. Majumdar, U. Rütt, O. Gutowski, M. V. Zimmermann, and S. Giri, Phys. Rev. B 98, 014408 (2018).

[37] A. Indra, K. Dey, A. Midya, P. Mandal, O. Gutowski, U. Rütt, S. Majumdar, and S. Giri, J. Phys.: Condens. Matter 28, 166005 (2016).

[38] K. Dey, A. Karmakar, A. Indra, S. Majumdar, U. Rütt, O. Gutowski, M. V. Zimmermann, and S. Giri, Phys. Rev. B 92, 024401 (2015).

[39] D. Orobengoa, C. Capillas, I. Aroyo, and J. M. Perez-Mato, J. Appl. Crystallogr. 42, 820 (2009).

[40] S. T. Bramwell, S. G. Carling, C. J. Harding, K. D. M. Harris, B. M. Kariuki, L. Nixon, and I. P. Parkin, J. Phys.: Condens. Matter 8, L123 (1996).

[41] A. Ghosh, K. Dey, M. Chakraborty, S. Majumdar, and S. Giri, Europhys. Lett. 107, 47012 (2014).

[42] A. Ghosh, A. Pal, K. Dey, S. Majumdar, and S. Giri, J. Mater. Chem. C 3, 4162 (2015).

[43] N. A. Benedek and C. J. Fennie, Phys. Rev. Lett. 106, 107204 (2011).

[44] N. A. Benedek, A. T. Mulder, and C. J. Fennie, J. Solid State Chem. 195, 11 (2012).

[45] M. Liu, Y. Zhang, L.-F. Lin, L. Lin, S. Yang, X. Li, Y. Wang, S. Li, Z. Yan, X. Wang, X.-G. Li, S. Dong, and J.-M. Liu, Appl. Phys. Lett. 113, 022902 (2018).

[46] S. Yoshida, H. Akamatsu, R. Tsuji, O. Hernandez, H. Padmanabhan, A. Sen Gupta, A. S. Gibbs, K. Mibu, S. Murai, J. M. Rondinelli, V. Gopalan, K. Tanaka, and K. Fujita, J. Am. Chem. Soc. 140, 15690 (2018).

[47] K. Shimamoto, S. Mukherjee, S. Manz, J. S. White, M. Trassin, M. Kenzelmann, L. Chapon, T. Lippert, M. Fiebig, C. W. Schneider, and C. Niedermayer, Sci. Rep. 7, 44753 (2017). 
[48] C. Dubourdieu, J. Bruley, T. M. Arruda, A. Posadas, J. JordanSweet, M. M. Frank, E. Cartier, D. J. Frank, S. V. Kalinin, A. A. Demkov, and V. Narayanan, Nat. Nanotechnol. 8, 748 (2013).

[49] H.-J. Liu, C.-W. Liang, W.-I. Liang, H.-J. Chen, J.-C. Yang, C.-Y. Peng, G.-F. Wang, F.-N. Chu, Y.-C. Chen, H.-Y. Lee, L. Chang, S.-J. Lin, and Y.-H. Chu, Phys. Rev. B 85, 014104 (2012).

[50] C. Daumont, W. Ren, I. C. Infante, S. Lisenkov, J. Allibe, C. Carrétéro, S. Fusil, E. Jacquet, T. Bouvet, F. Bouamrane, S. Prosandeev, G. Geneste, B. Dkhil, L. Bellaiche, A. Barthélémy, and M. Bibes, J. Phys.: Condens. Mater 24, 162202 (2012).
[51] D. H. Kim, H. N. Lee, M. D. Biegalski, and H. M. Christen, Appl. Phys. Lett. 92, 012911 (2008).

[52] H. N. Lee, S. M. Nakhmanson, M. F. Chisholm, H. M. Christen, K. M. Rabe, and D. Vanderbilt, Phys. Rev. Lett. 98, 217602 (2007).

[53] H. J. Zhao, L. Bellaiche, X. M. Chen, and J. Íñiguez, Nat. Commun. 8, 14025 (2017).

[54] Y. Shimakawa, Y. Kubo, Y. Nakagawa, S. Goto, T. Kamiyama, H. Asano, and F. Izumi, Phys. Rev. B 61, 6559 (2000).

[55] K. Dey, A. Indra, A. Chatterjee, S. Majumdar, U. Rütt, O. Gutowski, M. V. Zimmermann, and S. Giri, Phys. Rev. B 96, 184428 (2017). 